\title{
MIDIENDO DECISIONES LOCACIONALES. UNA APROXIMACIÓN A LA EVALUACIÓN DE LA DEFENSIBILIDAD EFECTIVA DE SITIOS ARQUEOLÓGICOS FORTIFICADOS
}

\author{
MEASURING LOCATIONAL DECISIONS. AN ASSESSMENT OF THE \\ EFFECTIVE DEFENSIVENESS OF FORTIFIED ARCHAEOLOGICAL SITES
}

\author{
César Parcero-Oubiña \\ Instituto de Ciencias del Patrimonio, Consejo Superior de Investigaciones Científicas. San Roque, 2, 15704 Santiago \\ de Compostela, España. \\ E-mail: cesar.parcero-oubina@incipit.csic.es
}

Presentado el: 24/10/2012 - Aceptado 10/07/2013

\begin{abstract}
Resumen
La fortificación de los espacios de asentamiento es un fenómeno ampliamente extendido en el registro arqueológico. Determinar la función y sentido de esa fortificación es a menudo un elemento clave en la caracterización social y política de los contextos en los que ocurre. En este trabajo se presenta un procedimiento de análisis basado en el empleo de SIG que, sobre una base mensurable, aporta elementos para la evaluación de las decisiones locacionales y constructivas que puedan estar detrás de la fortificación de los asentamientos. Se parte de plantear dos hipótesis principales: que la construcción de las estructuras defensivas siga una lógica efectivamente defensiva, o una monumental. Se definen una serie de factores mensurables que puedan relacionarse con ambas hipótesis: dónde y cómo, dentro de un poblado, sería esperable ubicar las defensas si se buscase un efecto defensivo, y dónde y cómo si se buscase un efecto monumentalizador. A través de un caso de estudio en un poblado de la Edad del Hierro en Galicia (NW de España) se muestra una implementación de este procedimiento, y la manera en la que se ha considerado la medición de variables que permitan responder a esas preguntas. El objetivo del trabajo es presentar la lógica y los detalles de un procedimiento analítico fácilmente aplicable con herramientas SIG estándar, antes que explorar a fondo las posibilidades interpretativas que se derivan de sus resultados.
\end{abstract}

Palabras claves: Asentamientos fortificados; Análisis locacional; SIG; Galicia 


\begin{abstract}
Settlement fortification is a widely extended feature in the archaeological record. The assessment of the meaning and function of fortifications is usually a key issue for the social and political characterization of the contexts where it occurs. This paper develops a GIS-based analytical procedure which aims at exploring and assess, in a measurable way, the locational and constructive decisions behind the fortification of settlement areas. At the onset, two main hypotheses are formed: the construction of settlement defenses is made according to the logic of monumentality, or to an actual logic of defense. Some measurable variables are explored that can be linked to either hypothesis: where and how defenses should be expected to occur within a specific settlement if they are aimed at a defensive function, and where and how if aimed at a monumental role. A case study, an Iron Age settlement in Galicia (NW Spain), illustrates the applicability of this procedure, and the way in which the variables considered are weighted. The paper focuses on the discussion of the foundations and practical details of an analytical procedure that is easily reproducible with any available GIS software, rather than on the exploration of the interpretative issues raised by the results.
\end{abstract}

Keywords: Fortified settlements; Locational analysis; GIS; Galicia

\title{
Introducción ${ }^{1}$
}

\section{Los sentidos de la fortificación}

La fortificación del asentamiento, o la creación de paisajes fortificados, es una forma de materialidad arqueológica de alcance global y transcultural. Son múltiples los contextos arqueológicos caracterizados por este tipo de elemento material, que típicamente ha sido manejado como una de las evidencias más representativas del conflicto y la guerra (p.e., entre otros muchos, Guilaine y Zammit 2002; Arkush y Ellen 2006). Sin embargo, si durante mucho tiempo pareció obvia y no discutible esta asociación, el desarrollo de lecturas alternativas para entender la lógica de estas "construcciones defensivas" ha venido adquiriendo gran fuerza en las últimas décadas, al menos en el contexto de la arqueología europea (Armit 2007)². De forma ante todo ilustrativa, se han planteado dos maneras esenciales de entender la lógica de esas fortificaciones, "militar" y "simbólica" (como resumen Armit 2007; Ruíz Zapatero 2012: 97-99). Para la primera, las fortificaciones serían construidas con una finalidad práctica defensiva. Para la segunda serían construcciones más asociadas con nociones como identidad, presencia, control o delimitación; ideas relacionadas con el concepto de monumento (sensu Criado Boado 1993). En gran medida, esta reformulación ha estado relacionada con un cuestionamiento más amplio de la relevancia de la guerra y el conflicto como mecanismos de relación social y política.

Aunque la discusión es mucho más amplia, una cuestión clave en ella es la de cómo de defensivas son las estructuras defensivas de los sitios. En contraste con lo central que esta cuestión parece ser, "most interpretations of architectural defensiveness are based on inferential logic, supported by comparison to a population of sites and independent lines of data which suggest that other sites with these traits are part of a history of war" (Martindale y Supernant 2009: 193). Salvo excepciones en las que se han abordado las propiedades efectivamente defensivas de las estructuras en función de su morfología (Keeley et al. 2007), las argumentaciones se han basado más habitualmente en atender a otras dimensiones del registro para rastrear en ellas posibles evidencias de conflicto, y usarlas entonces para definir el papel de las defensas (síntesis p.e. en Armit 2007). 
El contexto arqueológico conocido como Edad del Hierro en el noroeste de la Península Ibérica es un buen ejemplo de este tipo de debates (visiones genéricas sobre este contexto en González García 2007, González Ruibal 2008). Aquí, las alternativas a la lógica defensiva de la fortificación han sido diversas, semejantes a las propuestas para otros contextos, incluyendo lecturas como (según resume F. González García): “neutralizar las posibles tendencias a la dispersión de la comunidad, conceder visibilidad a los poblados, convirtiéndolos en marcas visibles en el paisaje frente a comunidades vecinas, actuar como elementos que servian para definir el status de la comunidad y su importancia, riqueza y prestigio, etc. $y$, sobre todo, proteger a la comunidad del medio natural exterior claramente hostil y lleno de peligros reales o imaginarios" (2006: 141, con referencias).

Una parte importante de la cuestión consiste en determinar para qué se construyeron esas estructuras defensivas, cuáles son las decisiones que están detrás de la forma y ubicación de una serie de elementos materiales que hoy podemos observar en el paisaje. En este trabajo se propone una aproximación a esto, enmarcada en el análisis locacional, en línea con alguna otra experiencia similar y haciendo uso de las capacidades de modelización y repetición que caracterizan a los SIG.

\section{Midiendo la defensibilidad}

La finalidad planteada de evaluar decisiones locacionales y constructivas requiere disponer de un bagaje conceptual y metodológico que nos permita acceder a esas decisiones a partir de las evidencias observables en el terreno (Verhagen y Whitley 2012). Además, si lo que pretendemos es ofrecer una argumentación no simplemente retórica o discursiva, debemos conseguir que esa metodología nos permita ofrecer un soporte mensurable de las observaciones que hacemos.

La secuencia que he seguido se apoya en la cadena lógica que marcan los conceptos decisiones-criterios-factores (propuesta en Parcero Oubiña y Fábrega Álvarez 2006; una cadena similar es ofrecida en Verhagen y Whitley 2012). En el caso que nos ocupa, la decisión que se quiere explorar es cuál es la lógica de las "estructuras defensivas" de un poblado, por qué se construyeron esas estructuras con una forma, dimensiones y localización concretas y no con otras posibles.

Concretar esas decisiones en criterios supone proponer diferentes argumentos posibles que hayan podido ser manejados en la toma de las decisiones que queremos recomponer. En el caso que nos ocupa, la discusión esquematizada en el apartado anterior nos proporciona dos posibles criterios a explorar: la defensibilidad y la monumentalidad. Se trata de plantear si las "estructuras defensivas" de un determinado poblado, su forma, dimensiones y localización, tienen sentido como resultado de una lógica efectivamente defensiva, si tienen sentido como monumentos, si ambos o si ninguno de ellos.

Finalmente, definir factores supone determinar cómo podemos detectar, y medir, en el terreno esos criterios: qué cosas observables y mensurables pueden ser empleadas como proxies respectivos de una lógica defensiva y de una lógica monumental, simbólica. Determinar estos factores nos permitirá formular hipótesis concretas que podamos testear contra la evidencia empírica. En el caso de este trabajo, los factores elegidos son extremadamente sencillos en su formulación. Como factor asociado a unos criterios defensivos se determina la accesibilidad/inaccesibilidad al lugar; esto es, la relación de 
la ubicación, forma y dimensiones de las estructuras artificiales con el incremento de la facilidad de acceso al asentamiento. Como factor asociado a unos criterios monumentales se determina la visibilización; esto es, la relación de la ubicación, forma y dimensiones de las estructuras artificiales con la transmisión de un mensaje a través de la posibilidad de que sean vistas de manera señalada ${ }^{3}$.

La posibilidad de cuantificar la defensibilidad no es nueva, y en parte lo que se propone converge, pese a haber sido desarrollado de forma autónoma ${ }^{4}$, con el planteamiento hecho por Martindale y Supernant (2011). No obstante, a diferencia de éste, que además es decididamente más sofisticado, el procedimiento aquí seguido está pensado más bien para la evaluación de sitios de manera singular, o al menos no exige una secuencia de casos para dotar de significación a los resultados. Esto no significa que sea una propuesta reconstructiva y positiva, que busque definir qué cosa es la defensibilidad y como reconocerla. Al contrario, es también una aproximación radicalmente relativa, basada en una lógica comparativa, cuya única asunción inicial es que los habitantes de un lugar decidieron, de forma consciente y de acuerdo con algunos criterios concretos, la construcción de una serie de estructuras artificiales del tipo murallas, fosos, parapetos, etc. Como resultado no se llega a una determinación absoluta de cómo de defensivo es un sitio, sino a la determinación relativa de cuáles de los factores que se formulan en forma de hipótesis permiten entender mejor la manera en la que un sitio singular se fortificó.

\section{El caso de estudio}

\section{El sitio}

Para poder explorar estas cuestiones, es necesario disponer de un caso de estudio que cumpla las siguientes condiciones:

- Ser un sitio fortificado, un lugar dotado de estructuras artificiales cuya posible funcionalidad fuese defensiva: fosos, murallas, parapetos.

- Que estos elementos sean perceptibles en superficie, siendo posible identificarlos con un trabajo de revisión superficial, no invasivo.

- Disponer de un conocimiento detallado del sitio como para poder tomar en cuenta, o desestimar, el posible efecto de una conservación desigual de diferentes áreas del sitio.

- Finalmente, disponer también de una documentación geométrica del sitio y su entorno con un detalle suficiente como para poder explorar las hipótesis que se están planteando.

El lugar elegido es el asentamiento fortificado de Castrolandín. Este sitio ha sido intensamente trabajado en el marco de un notable proyecto de recuperación patrimonial y arqueología pública (Ayán 2002; Otero y Porto 2007), que también ha incorporado la excavación de una parte importante del sitio (Criado y Ayán 2006; Ayán et al. 2007). Esto permite, para los fines de este trabajo, disponer no sólo de una detallada información superficial, sino también de un conocimiento sobre el estado de conservación, la relación entre lo hoy visible y lo originalmente construido, y sobre las modificaciones en la topografía del lugar ocasionadas por la construcción de las diferentes estructuras artificiales. 


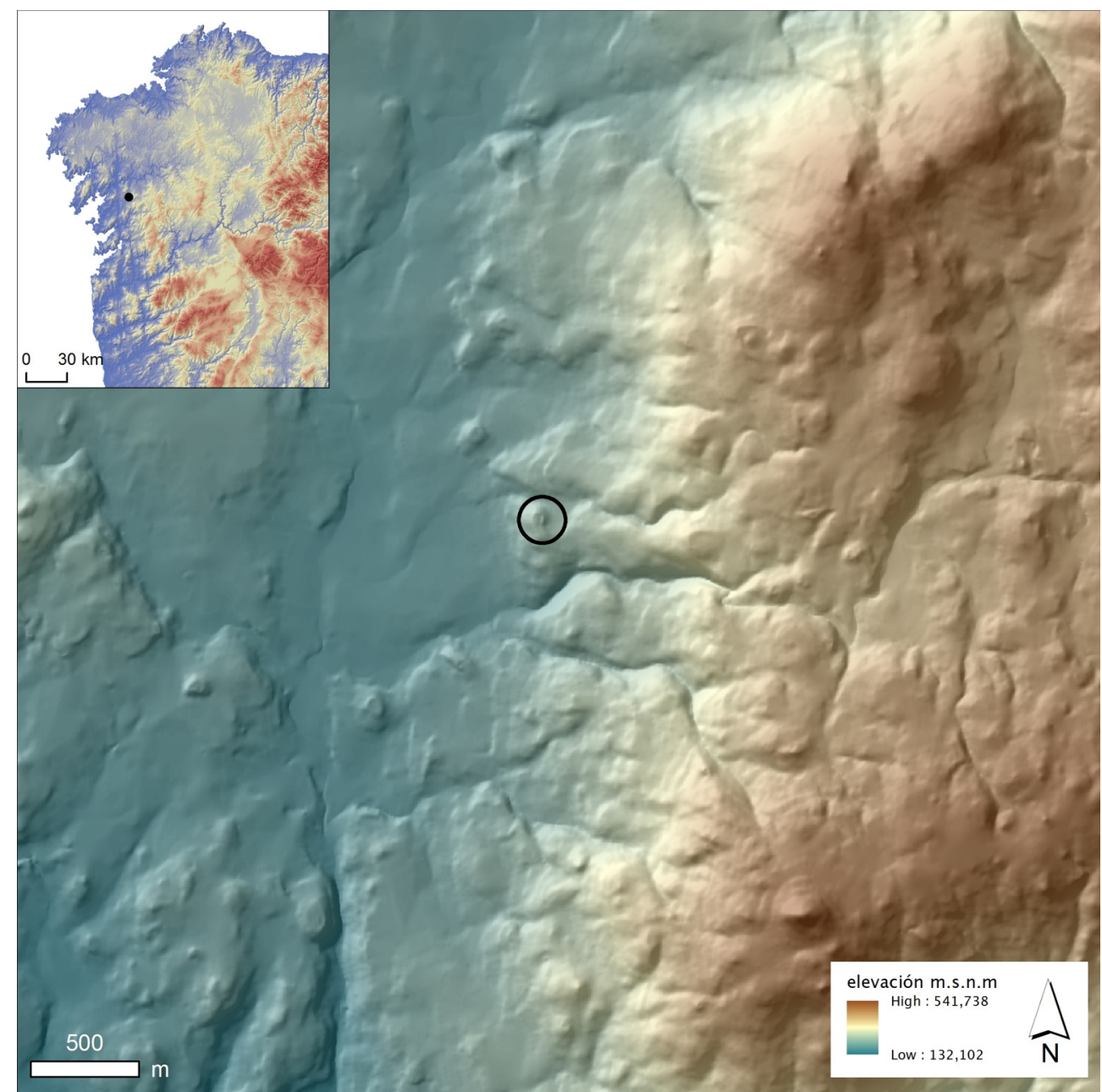

Figura 1. Localización del sitio de Castrolandín en el contexto del NW de la Península Ibérica y en su contexto local.

Castrolandín se localiza en las inmediaciones de la villa de Cuntis, en el sudoeste de Galicia (Figura 1). Se trata de lo que en el registro regional se conoce como castro, esto es, un pequeño asentamiento fortificado, que es la forma característica de asentamiento en la Edad del Hierro regional. A partir de los trabajos realizados (Figura 2) se ha fijado su secuencia de ocupación hacia finales de la Edad del Hierro y a inicios de la época romana (ca. siglos II BC y I AD). La secuencia de ocupación del sitio muestra dos fases principales de ocupación y uso, que se diferencian por la superposición de distintas estructuras habitacionales. Aunque internamente esos cambios están reflejando modificaciones notables en las formas del espacio doméstico (Ayán et al. 2007), no parecen haber supuesto un cambio en la morfología externa (extensión, estructuras defensivas o accesos), que sería resultado de un único proceso constructivo inicial.

El poblado ocupa una extensión de algo más de 1Ha., incluyendo la totalidad de estructuras artificiales actualmente visibles, aunque el espacio propiamente habitacional es de alrededor de $1 / 2 \mathrm{Ha}$. Su morfología es muy característica de los poblados de la llamada 


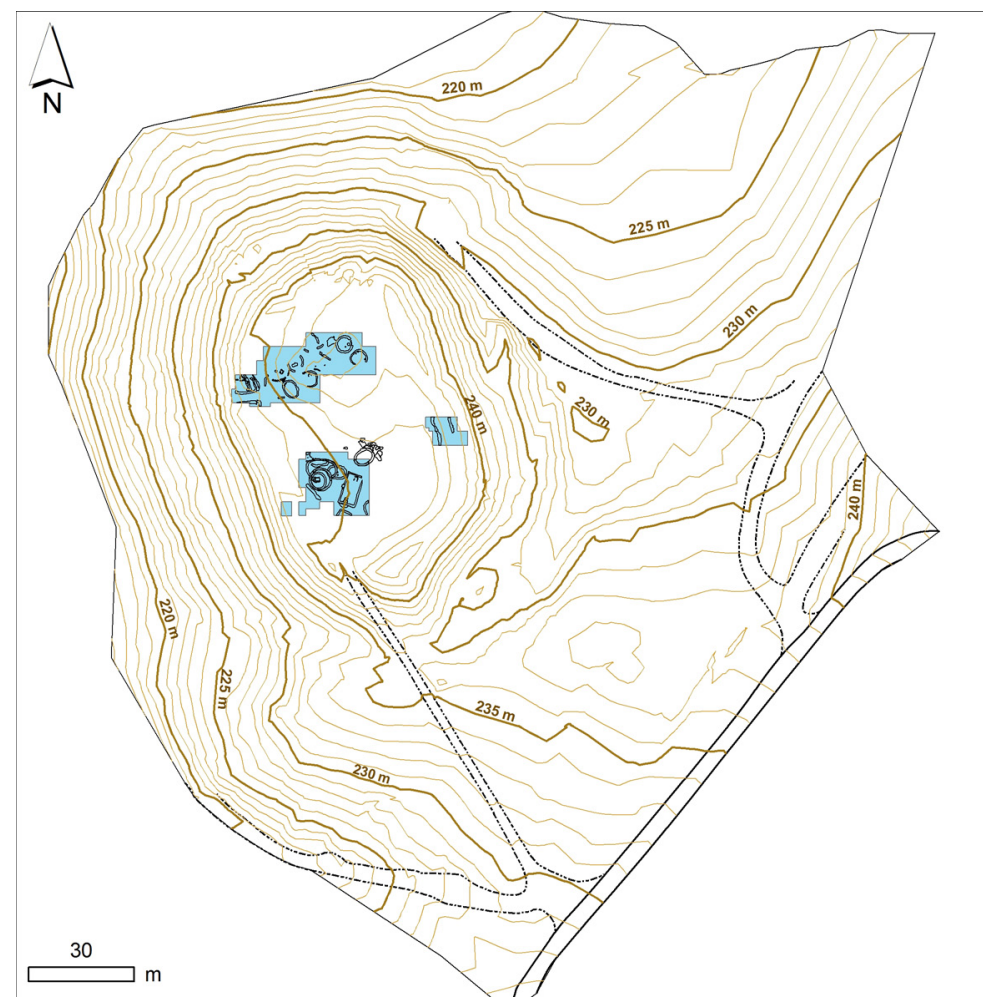

Figura 2. Visualización de la topografía actual de Castrolandín, con la localización de los sectores excavados y las principales estructuras documentadas. El camino de entrada al sur es moderno y corta las estructuras defensivas originales. El área al nordeste aparece suavizada por la menor densidad de datos topográficos disponibles debido a la cubierta vegetal.

Segunda Edad del Hierro (Parcero Oubiña 2000): posición en un cerro ligeramente elevado cercano a tierras bajas de valle, espacio habitacional delimitado y protegido por la propia topografía hacia el Oeste y por la construcción de una muralla de tierra complementada con al menos un foso exterior hacia el Este y Sur (Figura 2, ver también Figura 9).

Los datos obtenidos en las sucesivas campañas de trabajo realizadas en el sitio han permitido documentar un grado homogéneo de degradación de los volúmenes originales de las estructuras de delimitación. La muralla ha perdido algo de altura, los fosos se han colmatado parcialmente, pero no se ha observado que esto haya pasado con mayor intensidad en algunas zonas que en otras. Esto, como luego veremos, es importante considerarlo para entender la validez de las opciones analíticas que se han tomado.

\section{Datos manejados}

Para el conjunto de análisis que se muestran a continuación, los únicos datos necesarios son sendas representaciones de la topografía del sitio y de su entorno. La topografía del entorno se manejó a través de un modelo digital de elevaciones de 5 metros de resolución, realizado por interpolación en ArcGIS 9.2 a partir de los datos altimétricos de la cartografía vectorial 1:5000 de la Xunta de Galicia ${ }^{5}$. 
La topografía de detalle del sitio se obtuvo a partir de una selección de los datos procedentes de un escaneado láser 3D realizado con un equipo Leica HDS 3000 (el proceso de trabajo es uno de los ejemplos descritos en Mañana Borrazás et al. 2009: 43-47). En concreto, se usaron alrededor de 170.000 puntos, a partir de los cuales se construyó un TIN en ArcGIS $10.1^{6}$.

\section{Metodología y procedimiento de análisis}

Lo que se busca es, pues, explorar las diferentes decisiones constructivas adoptadas en la conformación de este poblado, a través de los factores expuestos más arriba. El problema puede ser expuesto en los siguientes términos: analizar la relación de una variable dependiente (la cantidad de trabajo invertido en la construcción de estructuras artificiales en la delimitación de cada una de las partes del asentamiento) con dos variables independientes (las condiciones topográficas de inaccesibilidad y de visibilización de cada una de esas partes). Para explorar esas relaciones, es necesario cuantificar cada una de las variables de alguna forma. En los apartados que siguen se irá viendo cómo he considerado esa medición.

Sin embargo, antes de ello es necesario abordar una tarea previa. Lo que interesa es ver cómo varían las decisiones constructivas a lo largo del perímetro del poblado. Por ello, es necesario segmentar ese continuo, el perímetro del poblado, en diferentes secciones discretas,

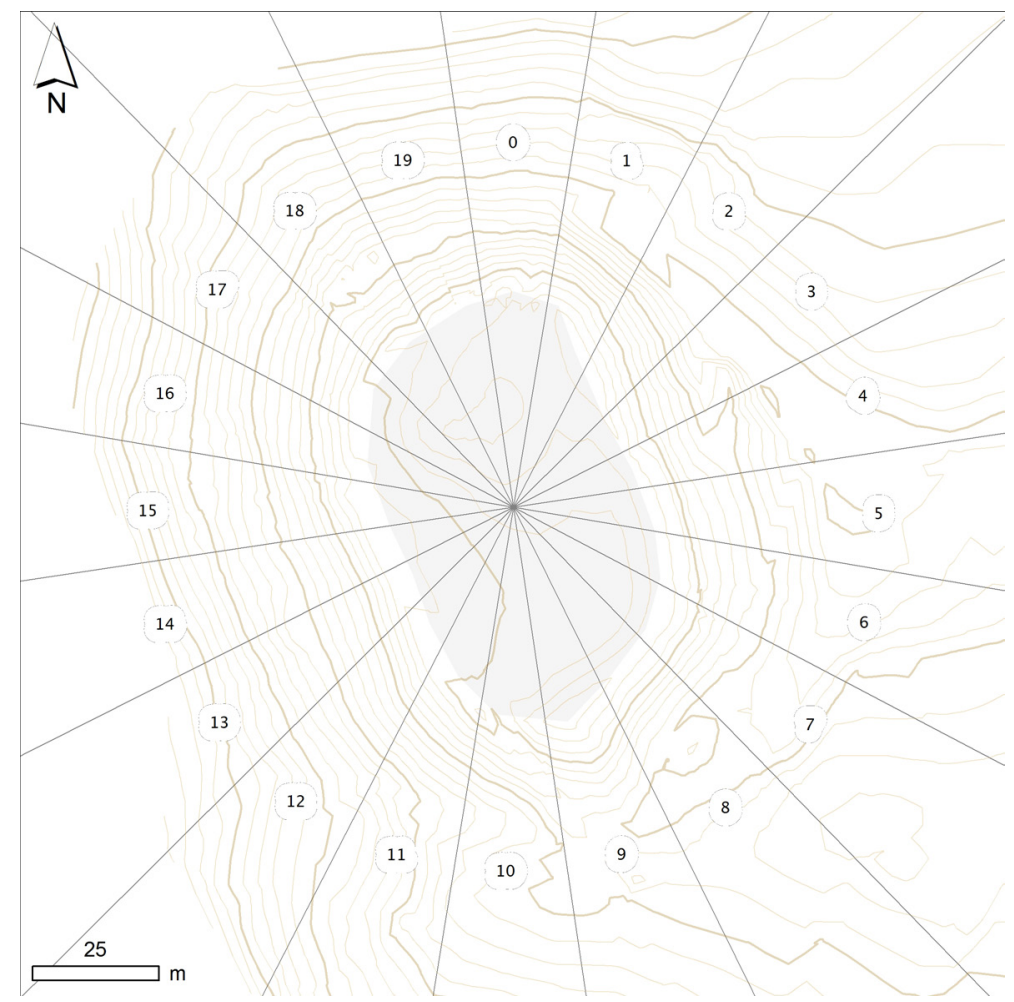

Figura 3. Resultado de la división del asentamiento en 20 sectores equivalentes como base para los diferentes análisis realizados. A partir de un punto central (centroide) del polígono que señala el recinto habitacional del castro (sombreado), se establecieron 20 sectores radiales. Los números identifican a cada sector. 
de manera que sea posible medir cada una de las variables en cada una de esas partes para poder después compararlas. Además, para que la comparación se efectiva es necesario que esas partes sean equivalentes.

El procedimiento seguido fue ubicar un punto en el centro del asentamiento y generar esos sectores de forma radial equidistante a partir de él (Figura 3). Dado que el polígono que representa la planta del asentamiento no es una figura geométrica regular, no tiene propiamente un centro, sino un centroide (el centro de masa del polígono que define la extensión del sitio, sombreado en la figura 3). Éste fue el punto a partir del cual se generaron los sectores de trabajo. Para decidir la cantidad de segmentos se tomó como referencia la anchura que estos sectores tomaban a la altura del perímetro exterior del área de asentamiento (la zona sombreada en la Figura 3). Se buscó un equilibrio entre generar sectores demasiado grandes, que no permitiesen un análisis detallado de cómo va variando el perímetro constructivo del poblado, o demasiado pequeños, que generasen una excesiva cantidad de datos en el proceso de análisis. Finalmente se crearon 20 sectores que, a la altura del límite exterior de la zona de asentamiento, tienen una anchura promedio de unos 11 metros.

Una alternativa a este procedimiento hubiera sido ubicar esos puntos en el perímetro de manera equidistante. Esto garantizaría una mayor "equivalencia" en algunos niveles (por ejemplo, para el análisis de visibilidad o de accesibilidad que luego se presentan), pero podría ocasionar un mayor desequilibrio de superficies a la hora de medir la variable que definimos como "inversión en construcción de estructuras artificiales". Es por esto que he optado por este procedimiento para establecer los sectores de análisis.

Usando estos sectores como unidades individuales de análisis, se procedió a cuantificar cada una de las tres variables propuestas (visibilización, inaccesibilidad y volumen de estructuras artificiales) en la forma en que a continuación se detalla.

\subsection{Determinación de la visibilización diferencial a lo largo del asentamiento}

Lo que se pretende explorar en este ámbito es cómo varía la forma en la que el castro es visible a lo largo de su perímetro, qué sectores del perímetro son más visibles que otros. Para ello, el procedimiento seguido fue muy sencillo: establecí un conjunto de 20 puntos a lo largo de las zonas más elevadas del perímetro del poblado, correspondientes a esos 20 sectores establecidos de partida (Figura 4). Para cada uno de los puntos, se realizó un cálculo de la cuenca visual potencial (viewshed) y se cuantificaron los resultados en diferentes intervalos de distancia (visibilización desde el entorno inmediato de $700 \mathrm{~m}$, y desde la larga distancia, hasta $10 \mathrm{~km}$ ). El uso de dos intervalos de distancia obedece a la intención de comprobar cómo de consistentes son las posibles diferencias en la visibilización según estemos cerca o lejos del sitio. El intervalo de $700 \mathrm{~m}$ se ha elegido, de manera algo convencional, para representar el entorno inmediato del sitio, desde el cual sería posible la percepción diferenciada de un elemento como la arquitectura. Intervalos similares se vienen usando para acotar el "entorno visual inmediato" en diferentes campos, desde la arqueología (Sevenant y Antrop 2007) a la arquitectura (Otero et al. 2009). El intervalo de $10 \mathrm{~km}$ representa, en función de la extensión del área de trabajo, la visibilización potencial total del sitio desde cualquier lugar considerado. 


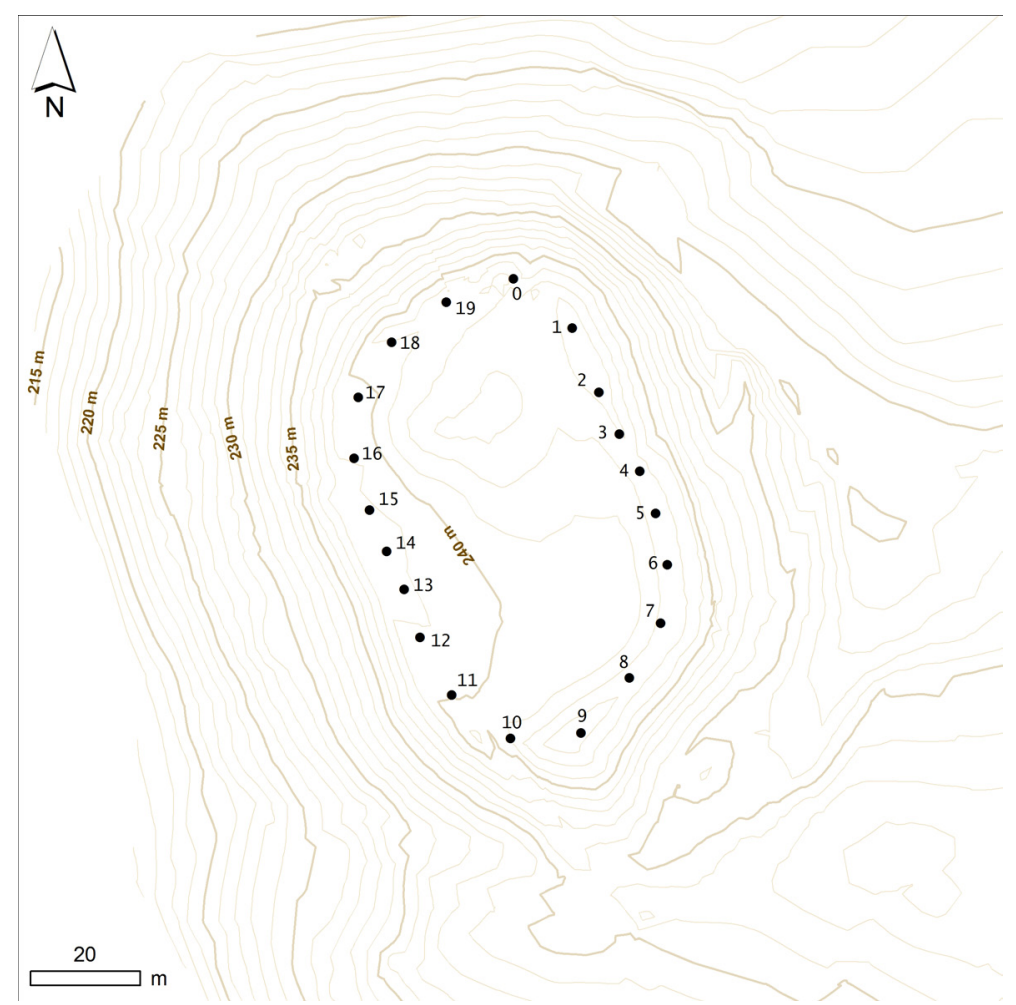

Figura 4. Localización de los 20 puntos empleados para el análisis de la visibilización del perímetro del asentamiento. Cada punto se localiza en el eje de uno de los sectores indicados en la Figura 3. Obsérvese cómo se localizan en las partes más elevadas en cada sector, para obtener como resultado la mayor visibilización potencial posible en cada caso.

Para realizar esta determinación se usó el antes descrito DEM del entorno como representación de la topografía y, por tanto, de las condiciones potenciales de conexión visual entre cada lugar del entorno y los puntos de referencia. Además, y dado que lo que se pretende analizar es cómo se observan desde fuera cada una de las partes del sitio, se añadió a cada uno de los puntos del entorno una elevación de 1,7 metros para representar la estatura media de un potencial visor, mientras que los puntos ubicados sobre el perímetro del sitio se mantuvieron con la elevación a ras del terreno.

La cuantificación de los resultados permite establecer cómo de visibles desde el entorno son las diferentes posiciones, cuáles lo son más y menos. Para expresar esta variabilidad, cuantifiqué la superficie total de terreno del entorno $\left(\mathrm{en}^{\mathrm{km}}{ }^{2}\right.$ ) desde la que cada uno de los sectores concretos es potencialmente visible. El resultado final ofrece una ilustrativa comparativa (Figura 5), que muestra que efectivamente hay porciones del perímetro más visibles que otras, entendiendo por ello que se pueden ver desde más lugares.

Podría ocurrir que el entorno inmediato estuviese muy condicionado por variaciones locales en la topografía. Entonces, podemos ver cómo de potencialmente visibles son los sectores desde la larga distancia, de modo que esas diferencias locales no afecten. Para 


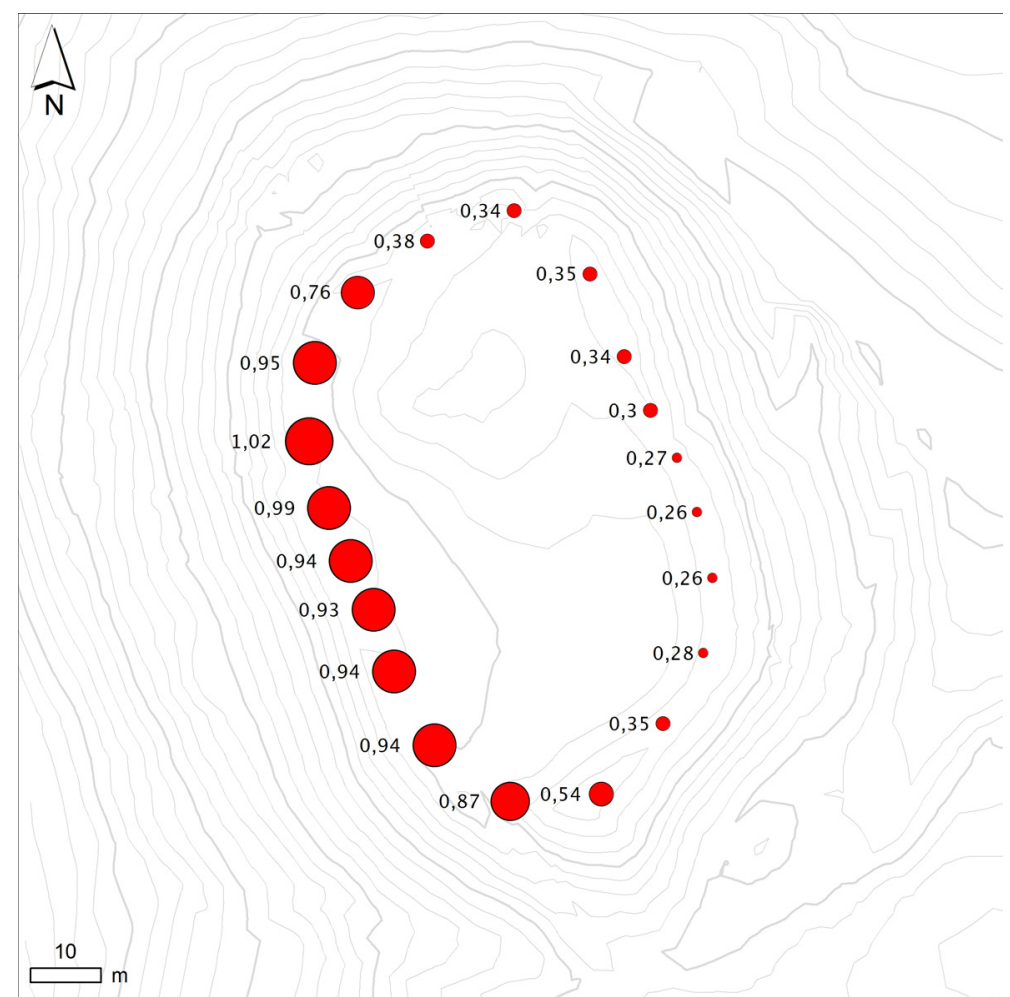

Figura 5. Resultados del análisis de visibilización desde el entorno inmediato $(700 \mathrm{~m})$. Los valores asociados a cada punto expresan $\left(\mathrm{en}_{\mathrm{km}}^{2}\right.$ ) desde cuántos lugares de ese entorno es potencialmente visible ese sector concreto. El tamaño del punto es proporcional a ese valor.

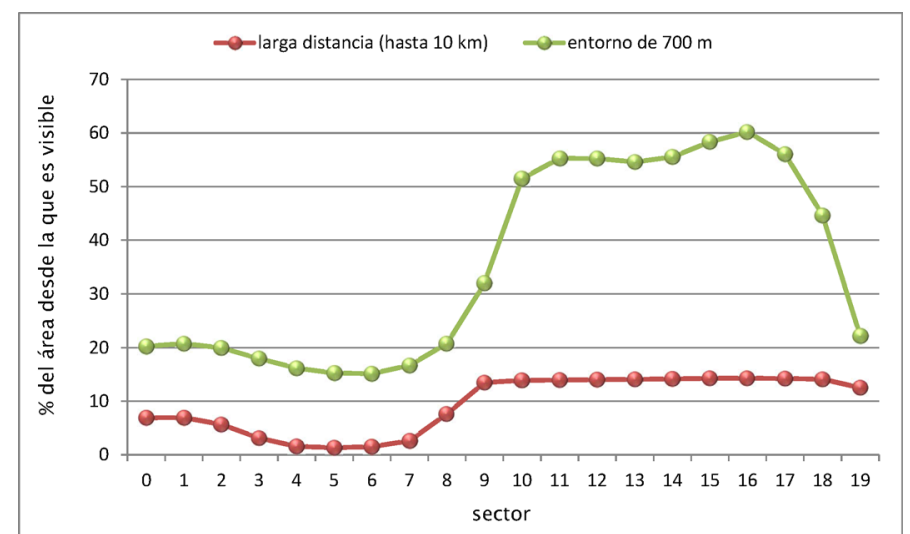

Figura 6. Comparación de la visibilización de los diferentes sectores del castro en el entorno inmediato $(700 \mathrm{~m})$ y a larga distancia (hasta $10 \mathrm{~km}$ ). Los valores representan el porcentaje del total del entorno desde el que cada sector es visible.

poder combinar de forma más comparable estos resultados, lo que se hizo no fue comparar directamente las cifras absolutas de superficie de terreno desde la que cada sector es visible, sino el porcentaje del total de terreno existente en esos dos intervalos de distancia desde el que existe conexión visual. El resultado (Figura 6) muestra que los valores relativos entre los diferentes sectores se mantienen constantes. Lógicamente hay una notable variación en las 
cifras (a medida que nos alejamos crece la proporción de posiciones desde las que el castro deja de verse). Pero las diferencias relativas se mantienen, lo cual es visible gráficamente en el hecho de que los ascensos y descensos de las líneas se producen en los mismos sectores.

En síntesis, esta parte analítica permite comprobar que hay un área del perímetro del asentamiento (al Oeste, que incluye los sectores 10 a 18) que tiene unas condiciones de visibilización muy superiores al resto. O sea, que esos sectores son visibles desde muchos más lugares del entorno del sitio. En el extremo contrario, los sectores que son visibles desde menos lugares del entorno son los 4 a 8 .

\subsection{Determinación de la accesibilidad diferencial a lo largo del asentamiento}

La segunda variable que se analiza es la inaccesibilidad al poblado. Dado que se ha formulado como variable independiente, lo que interesa es medir las condiciones topográficas de accesibilidad al lugar que ocupa el poblado. Por ello, lo que se ha medido es la dificultad para acceder a los pies de las estructuras construidas exteriores desde las diferentes direcciones que señalan los sectores que se delimitaron.

La cuantificación de esta variable se ha hecho mediante otra de las rutinas más habitualmente empleadas en el análisis espacial con SIG en arqueología: los cálculos de coste de desplazamiento (una presentación general por ejemplo en Connelly y Lake 2009). Estas

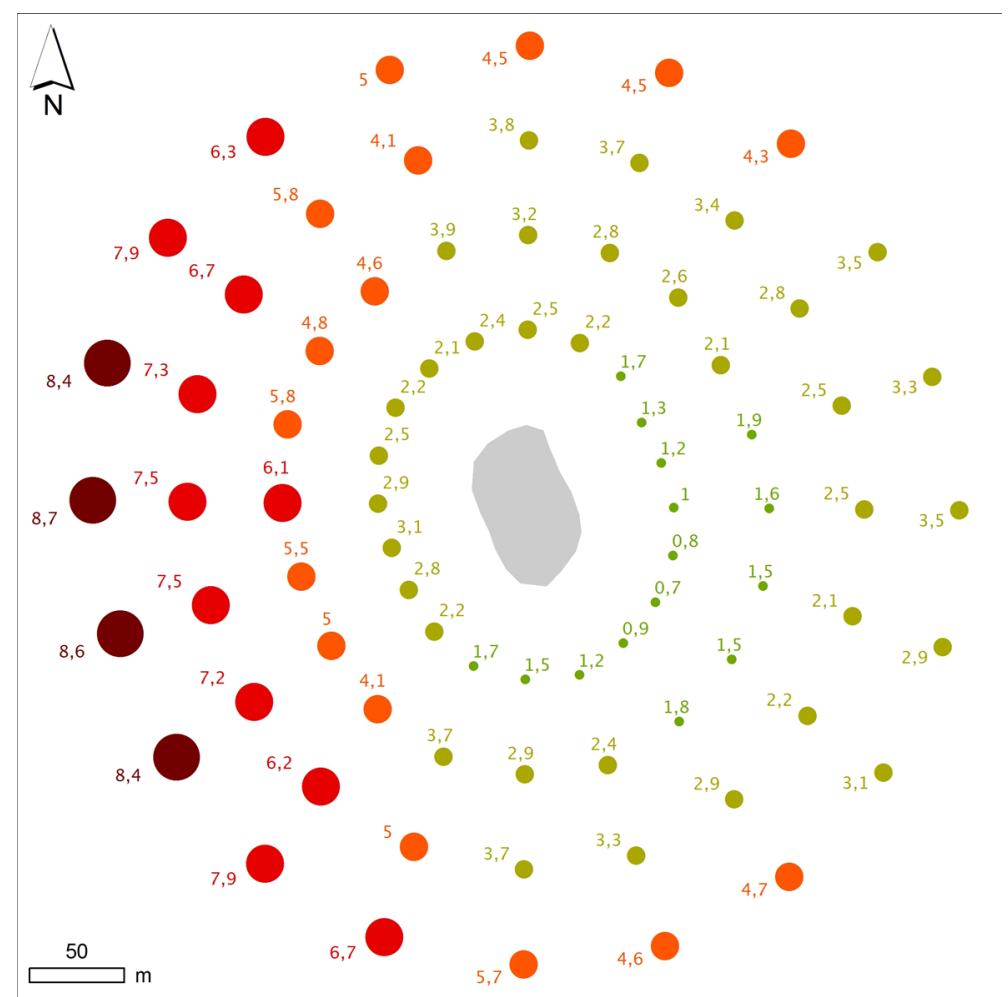

Figura 7. Ilustración de los resultados del análisis de accesibilidad a 50, 100, 150 y 200 metros lineales del castro. Los valores asociados a cada punto expresan el esfuerzo (en tiempo, minutos) necesario para alcanzar desde allí el perímetro exterior del asentamiento. Costes más altos indican más dificultad de acceso. El tamaño y tono del punto son proporcionales a ese valor. Cada punto se corresponde con uno de los sectores indicados en la Figura 3. 
determinaciones se basan en definir una serie de parámetros mensurables que condicionen el movimiento humano en el paisaje (por ejemplo, y típicamente, la pendiente del terreno) y construir un modelo matemático que permita medir cuánto lo afectan y, así, estimar de manera teórica para cualquier espacio el coste necesario para desplazarse entre dos lugares. Este coste es típicamente expresado en unidades como energía o tiempo. A partir de esta idea básica es posible realizar aplicaciones que aporten nuevo conocimiento sobre los patrones de movilidad en relación con sitios arqueológicos (p.e. Fairén 2004, Rahn 2005, Howey 2007, Murrieta Flores 2012) o proponer nuevas determinaciones más complejas y abstractas (p.e. Bevan 2011, Llobera et al. 2011).

En este caso, la determinación del coste de acceso se ha calculado empleando la pendiente del terreno como factor de coste, según los parámetros definidos en la ampliamente utilizada propuesta de W. Tobler (1993). Tomando como lugar de llegada el exterior de la totalidad de estructuras visibles en el sitio, se ha medido para cada sector el

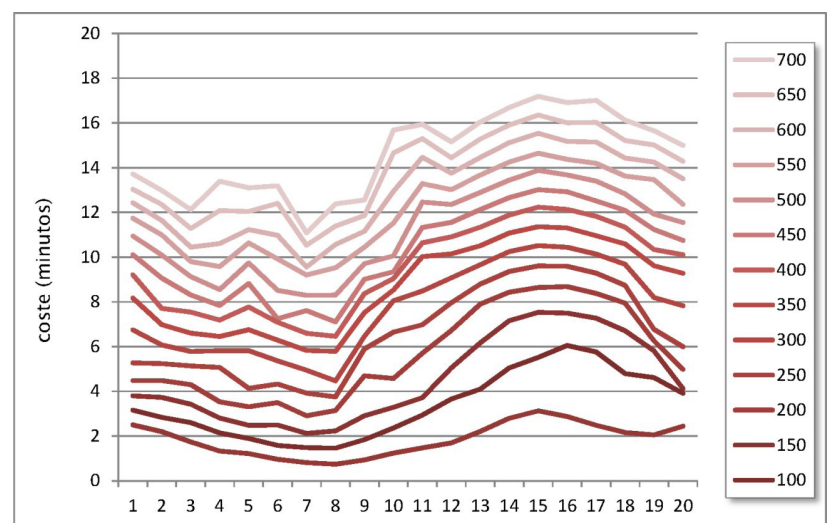

Figura 8. Comparación del coste de acceso al yacimiento. En la vertical, coste expresado en tiempo (minutos). En la horizontal, cada número se corresponde con uno de los sectores señalados en la Figura 3, e indica por tanto una misma dirección de aproximación al castro.

coste de desplazamiento desde una distancia lineal máxima de 700 metros, y a intervalos de 50 metros. Los resultados muestran (Figura 7, Figura 8) que de nuevo encontramos diferencias entre los sectores. En este caso muestran un acceso desigualmente costoso al sitio que informa de unas condiciones topográficas más asequibles para llegar a él desde el Este. El interés de mostrar la serie sucesiva de intervalos que se refleja en las figuras adjuntas es comprobar cómo las diferencias de coste de acceso que existen en el primer radio considerado $(50 \mathrm{~m})$ se mantienen a medida que nos alejamos del poblado (Figura 8). Esto proporciona robustez a los resultados e informa, efectivamente, de que esa diferente dificultad de acceso no obedece a factores puntuales sino que es consistente a lo largo de una misma dirección.

\subsection{Inversión en construcción de estructuras artificiales en las diferentes partes del asentamiento}

Finalmente, resta por cuantificar la tercera variable, la definida como dependiente: la diferente inversión de trabajo en la construcción de estructuras artificiales en la delimitación del sitio. Un simple vistazo a la topografía actual del castro permite apreciar que hay un mayor desarrollo de estas estructuras hacia el lado Este, donde se documenta una muralla y al menos un foso exterior a ésta. Por el contrario, hacia el Oeste en superficie no se aprecia 
más que un aterrazamiento que no conforma un volumen emergente. En los trabajos de excavación realizados en esta parte se documentaron restos de un muro de piedra muy desmoronado, pero que no habría alcanzado una altura comparable a la de la muralla al Este. De hecho, no hay evidencias de una pérdida de volumen desigual en las estructuras del poblado (com. pers. de Carlos Otero Vilariño, responsable de las últimas intervenciones; ver también Criado y Ayán 2006; Ayán et al. 2007).

En cualquier caso, lo que se necesita es una manera de poder cuantificar esa variación en la inversión de trabajo que se realizó en la construcción de estas estructuras en distintas partes del perímetro. En ausencia de un proceso real de experimentación directa sobre el terreno, una forma de aproximación a ello es medir qué cantidad de material se movilizó en la construcción de las murallas o aterrazamientos y en la excavación de los fosos. Es cierto que esto omite posibles diferencias de esfuerzo debidas a, por ejemplo, la eventual existencia de materiales de diferente dureza o peso en distintos lugares del sitio. No obstante, dado el reducido tamaño del poblado, y la uniformidad del componente geológico documentado en él, este factor puede considerarse menor.

Este propósito es, de nuevo, algo que la disponibilidad de herramientas digitales hace posible, ya que puede plantearse como una simple cuestión de cuantificación de la diferencia de volúmenes entre dos superficies. El problema estriba en que únicamente disponemos de una superficie, la actual, resultante ya de la construcción de las estructuras artificiales ${ }^{7}$. Lo que se necesita es una recreación de la posible apariencia original del cerro, antes de la construcción del poblado.

Este es un punto donde el trabajo encuentra problemas, de dos tipos. Por un lado, la información de que disponemos sobre esa apariencia original es dispersa, ya que procede de los datos extraídos de los sectores excavados y de aquellos puntos en los que en superficie aflora directamente la roca. Por otro lado, las herramientas disponibles para modificar superficies digitales (al menos aquellas a las que hemos podido acceder) se basan en una modificación manual, no orientada por la introducción de valores numéricos concretos. Por ello, el proceso que se siguió para recrear esa posible superficie original es únicamente aproximado y orientativo, aunque lo menos especulativo posible, ya que nos hemos limitado a eliminar las estructuras más obviamente artificiales. Empleando el software 3D Reshaper 6.0 se rebajaron las zonas ocupadas actualmente por la muralla y los aterrazamientos y se rellenaron los espacios ocupados por el foso exterior, manteniendo de manera casi exacta la cota actual del terreno tanto en el interior del poblado como en las laderas exteriores del cerro, donde no se han documentado alteraciones topográficas importantes.

El resultado (Figura 9) muestra una topografía reconstruida que es básicamente la de un cerro cónico achatado. Difiere de la actualmente visible esencialmente en las zonas en las que hay estructuras artificiales claramente visibles.

Una vez definidas ambas superficies, el proceso de determinación de las diferencias de volumen entre cada una de ellas es un cálculo sencillo, que una vez más se realizó de manera individualizada para cada sector. De este modo, podemos comparar el grado de alteración topográfica practicado en cada uno de ellos. En este caso, y antes de poder comparar los resultados, fue necesario aplicar una nueva corrección. Los sectores de trabajo tienen una superficie ligeramente diferente entre sí, debida a la irregularidad 


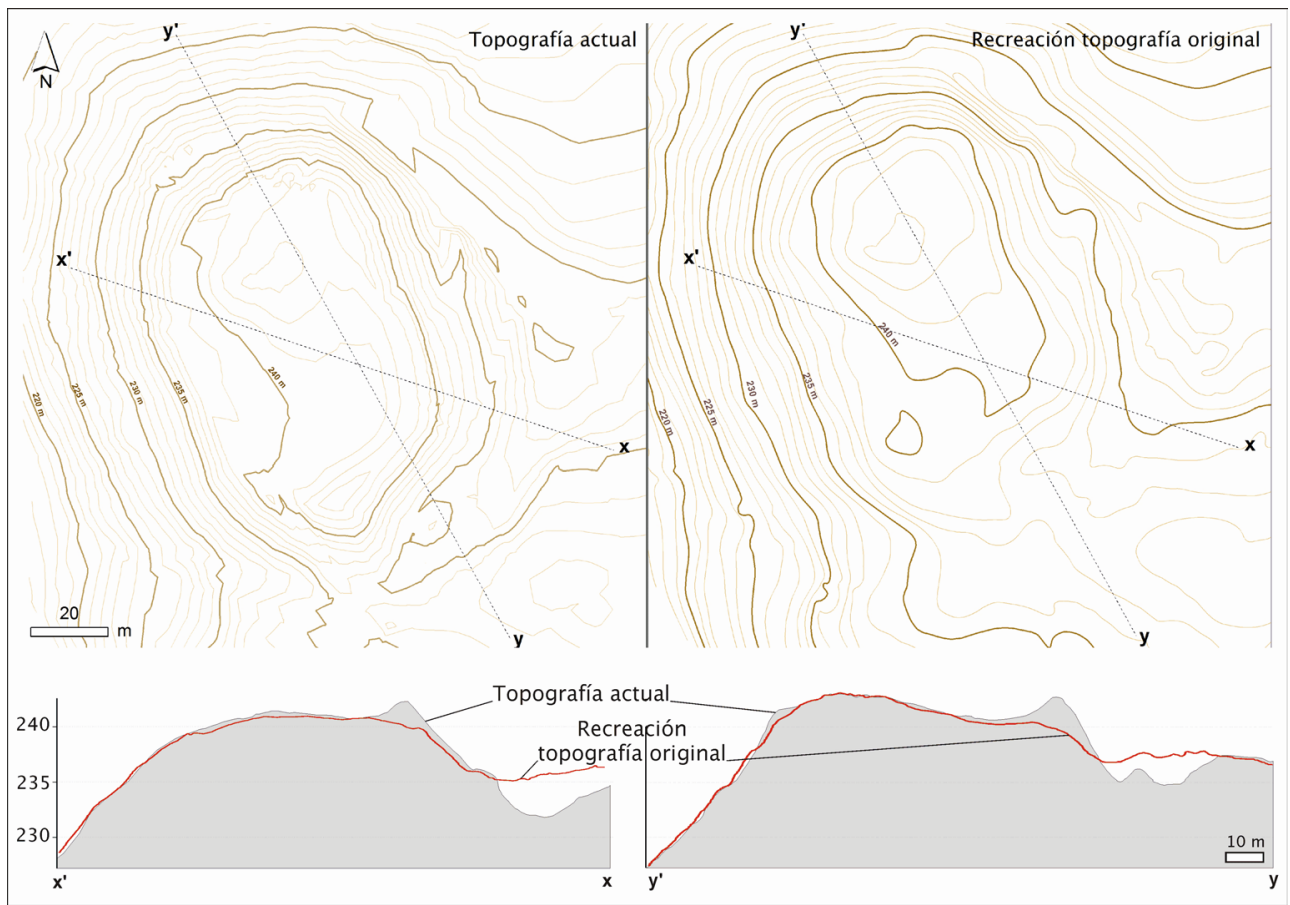

Figura 9. Topografía actual (izquierda) y recreación hipotética de la superficie original del cerro, previa a la construcción del asentamiento (derecha). Abajo, secciones topográficas actuales (sombreado) y secciones correspondientes en la recreación de la topografía original (línea superpuesta). Se observa cómo las mayores diferencias están en la muralla y los fosos, en el lado Este y Sur del cerro.

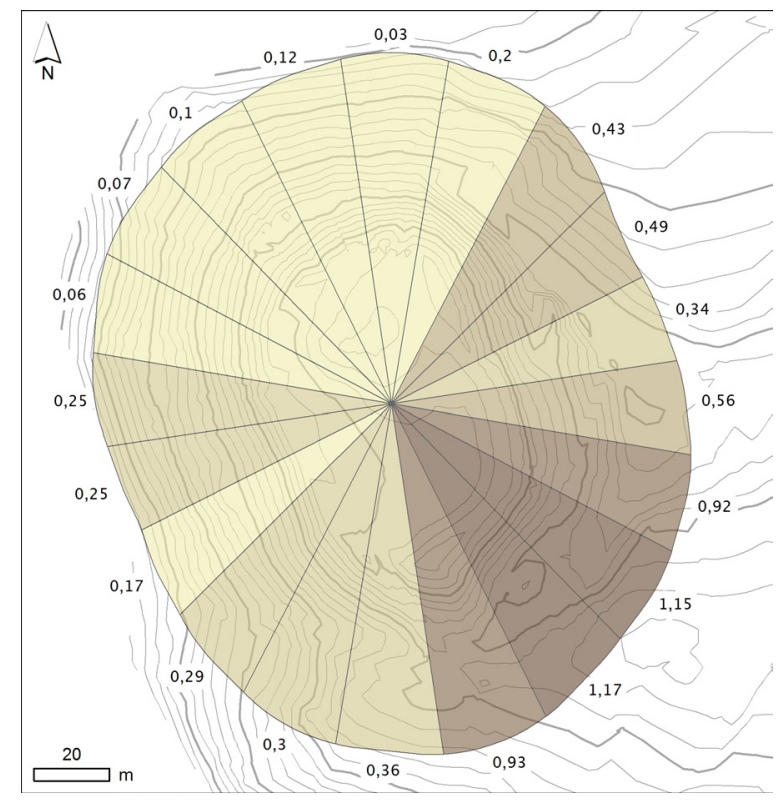

Figura 10. Resultado de la cuantificación de la cantidad de material movilizado en la construcción de estructuras artificiales. Las cifras representan el "volumen normalizado" $\left(\mathrm{m}^{3} / \mathrm{m}^{2}\right)$. El sombreado de los sectores es proporcional a ese valor. 
de la figura geométrica del sitio que sirve de base (Figura 10). Aunque esto no afectaba a los cálculos anteriores, en este caso sí hay que considerarlo. En efecto, ahora estamos comparando diferencias en volúmenes $\left(\mathrm{m}^{3}\right)$ alterados en cada sector, y es esperable que los sectores más extensos arrojen valores más elevados. Para poder compararlos de manera correcta, es necesario eliminar el efecto de las diferencias de superficie, generando un valor de "volumen normalizado", que se obtiene de forma muy sencilla:

Volumen normalizado $=($ volumen total modificado en el sector $) /($ superficie sector $)$

Este volumen normalizado de material removido (VNMR en adelante) expresa, pues, los $\mathrm{m}^{3}$ de material movilizados (añadidos o extraídos) por $\mathrm{m}^{2}$ de superficie. El resultado, como cabía esperar, muestra unos valores más elevados en la mitad Este y Sudeste del sitio. Ahora bien, el valor de estos resultados no es única ni principalmente el constatar una diferencia claramente perceptible en el sitio, sino el ofrecer una cuantificación, aunque sea aproximada, al valor concreto de esa diferencia. Esta cuantificación no pretende en absoluto ser exacta, sino simplemente ilustrativa de las diferencias relativas en la modificación del cerro a lo largo de su perímetro. Disponer de una cuantificación así es lo que va a permitir emplear esta variable para testar numéricamente una serie de hipótesis concretas, como a continuación se describe.

\section{Resultados}

\section{Evaluación de hipótesis planteadas}

Una vez determinados los resultados individuales de la medición de cada una de las tres variables consideradas, es posible testear las hipótesis de partida empleando un simple test de correlación. Las correlaciones varían en función de la hipótesis que se quiera testear, así que se presentan ordenadas de esta forma.

Hipótesis 1: La inversión de esfuerzo en el asentamiento mediante la construcción de estructuras artificiales está directamente relacionada con la defensa del lugar. Es esperable que haya una correlación positiva entre el esfuerzo invertido en la construcción de las estructuras y las condiciones defensivas del lugar.

Para verificar esta hipótesis, atenderemos a la correlación existente entre la variación en las condiciones topográficas de acceso al poblado y la inversión de esfuerzo en la construcción de estructuras artificiales a lo largo del perímetro. El resultado de esta correlación (medida mediante el coeficiente de Pearson) ${ }^{8}$ es el siguiente (para diferentes intervalos de distancia):

Pxy $($ coste a $50 \mathrm{~m}, \mathrm{VNMR})=-0,795$

Pxy $($ coste a $100 \mathrm{~m}, \mathrm{VNMR})=-0,707$

Existe, pues, una correlación negativa en los términos en los que se ha medido esta relación; es decir, según aumenta el coste de acceso al poblado (más dificultades topográficas), disminuye la inversión de esfuerzo en la construcción de estructuras artificiales. En otras palabras, las zonas donde más elementos artificiales se concentran son las de más fácil acceso. Además, esta correlación es elevada, relativamente próxima a 1, lo cual indica una tendencia a que, según disminuye la dificultad de acceso, aumenta en la misma proporción el volumen de estructuras artificiales. 
El resultado de este test permite, pues, sostener la hipótesis de que la construcción de estructuras artificiales sigue una lógica efectivamente defensiva. Aunque me detendré más en esto al final del trabajo, es importante señalar desde ahora que esta correlación, como cualquier otra, no supone necesariamente una relación de causalidad. Lo único que muestra es que hay una convergencia entre ambos factores, pero el entender o no a uno como causa del otro es sólo una de las posibles interpretaciones de esta correlación. Al tiempo, también es importante señalar que la existencia de esta correlación no excluye la eventual existencia de otras en las que se impliquen los mismos factores. Para ver si esto ocurre, podemos pasar a analizar una segunda hipótesis.

Hipótesis 2: La inversión de esfuerzo en el asentamiento mediante la construcción de estructuras artificiales está directamente relacionada con la monumentalización del lugar. Es esperable que haya una correlación positiva entre el tamaño de las defensas y las condiciones de visibilización del lugar, concentrando las defensas en las zonas más visibles del poblado.

Para verificar esta hipótesis, atenderemos a la correlación existente entre la variación en las condiciones de visibilización de cada parte del perímetro del poblado y la inversión de esfuerzo en la construcción de estructuras artificiales a lo largo del perímetro. El resultado de esta correlación es el siguiente (para diferentes intervalos de distancia):

Pxy (visiblización desde $700 \mathrm{~m}, \mathrm{VNMR})=-0,505$

Pxy (visiblización desde larga distancia, $\mathrm{VNMR})=-0,492$

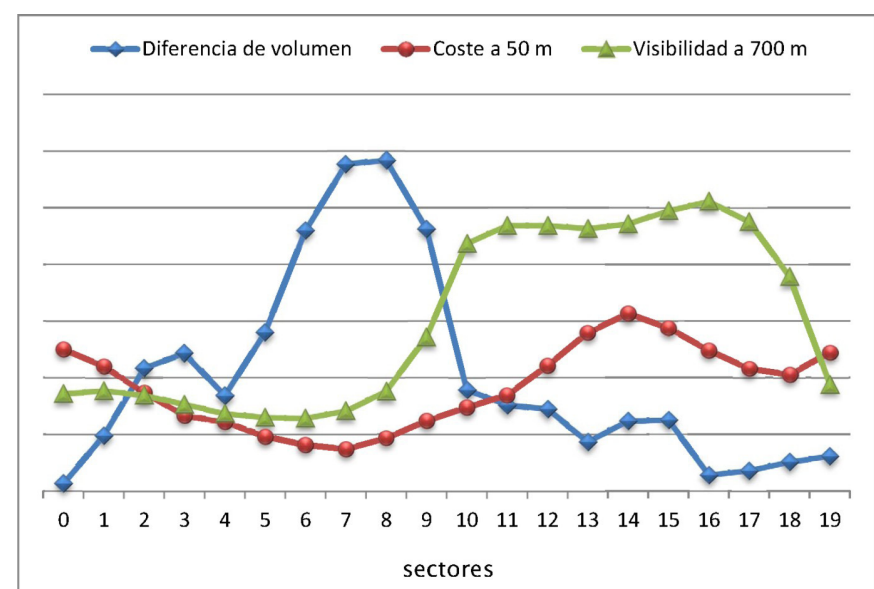

Figura 11. Relación de cambio entre las tres variables consideradas para cada sector: visibilización desde la corta distancia $(700 \mathrm{~m})$, coste de acceso desde la corta distancia $(50 \mathrm{~m})$ y volumen de obra artificial. No se muestran valores en el eje $\mathrm{Y}$ ya que cada variable está expresada en unidades diferentes.

Contra lo que cabría esperar, existe de nuevo aquí una correlación negativa en los términos en los que se ha medido esta relación; es decir, según aumentan las condiciones de visibilización de cada parte del poblado (visible desde más lugares), disminuye la inversión de esfuerzo en la construcción de estructuras artificiales. En otras palabras, las zonas donde más esfuerzo se ha invertido en la construcción de elementos artificiales no son las más visibles desde el entorno. Y esto ocurre tanto considerando el entorno más inmediato como el más distante. En este caso la fuerza de la correlación es algo menor, no hay una tendencia tan clara a que una cosa disminuya en función de la otra. Esta correlación puede observarse también gráficamente en la Figura 11. 
El resultado de este test no permite soportar la idea de que la construcción de las estructuras artificiales del poblado (muralla, fosos, etc) esté condicionada por una voluntad de visibilización. Si ése fuera el caso, sería esperable que las estructuras más conspicuas se distribuyesen en las partes más visibles del perímetro del poblado. Contra lo que ocurre con la correlación positiva anterior, en este caso sí es posible descartar que exista una relación de causalidad entre ambos factores, al menos en la manera en la que han sido considerados y medidos.

Ahora bien, sería posible argumentar que hay dos problemas con esta determinación. El primero sería que en este caso hemos medido más o menos lo mismo que en la primera hipótesis: las zonas más inaccesibles han de ser también las más visibles, ya que ambas dependen en gran medida de la existencia de pendientes más fuertes en el entorno. Aunque esto no es necesariamente una relación unívoca y universal (como demuestran los resultados de otro sitio analizado, que presentaremos en el apartado 3.3), será bueno intentar evaluar la diferente percepción del sitio desde el entorno de alguna otra forma.

El segundo posible problema es que no es adecuado medir la importancia de la visibilización únicamente de forma cuantitativa; puede ser más importante considerar el cómo se ve algo que el cuánto se ve. Si consideramos la percepción como algo cualitativo, deberíamos tener en cuenta muchos otros factores diferentes de la mera presencia o ausencia de obstáculos visuales entre nosotros y lo que pretendemos mirar. Este factor, la prominencia visual, es un campo donde se han realizado avances tanto desde el punto de vista puramente perceptivo (tal vez el más conocido es Higuchi 1983) como en la manera en la que puede ser modelizado y considerado computacionalmente en arqueología (Wheatley y Gillings 2000, o varios de los trabajos de M. Llobera, p.e. Llobera 2001, 2007). Obviamente para que exista una percepción ha de existir conexión visual, pero más allá de ello la manera en la que los objetos se nos aparecen, y el efecto que pueden generar en nosotros, varía en función de otros factores.

Si consideramos el caso de estructuras como las que nos ocupan, y asumimos, o tratamos de explorar, la idea de que hubiesen jugado un papel monumentalizador, podríamos indagar en la manera en la que esas estructuras son vistas. La relación topográfica entre el observador y lo observado puede manipular la percepción, generando efectos que hagan aparecer las cosas más grandes o pequeñas de lo que son en realidad. En concreto, me centraré aquí en un efecto: la aproximación visual a un objeto desde un punto inferior tiende a agrandar su tamaño, mientras que la adopción de un punto de vista elevado lo reduce. Y esto es algo que, aunque depende de que exista conexión visual, es independiente de la amplitud de esta: desde el cielo, o desde un punto elevado, la visibilidad de un paisaje es muy amplia cuantitativamente, pero la percepción de las cosas las empequeñece respecto a cómo se perciben desde el terreno o desde una cota baja, aunque en este caso la "cantidad de visibilidad" sea menor.

A partir de esta sencilla idea, es posible entonces plantear una tercera hipótesis, que permita no descartar todavía el papel de la percepción como factor locacional de las estructuras artificiales de los castros. La formulación sería:

Hipótesis 3: La inversión de esfuerzo en el asentamiento mediante la construcción de estructuras artificiales está directamente relacionada con la monumentalización del 
lugar. Es esperable que haya una correlación positiva entre el tamaño de las defensas y las condiciones de percepción del lugar, concentrando las defensas en las zonas del poblado hacia a las cuales un observador se aproximaría desde una posición inferior, lo que genera un efecto de agrandamiento.

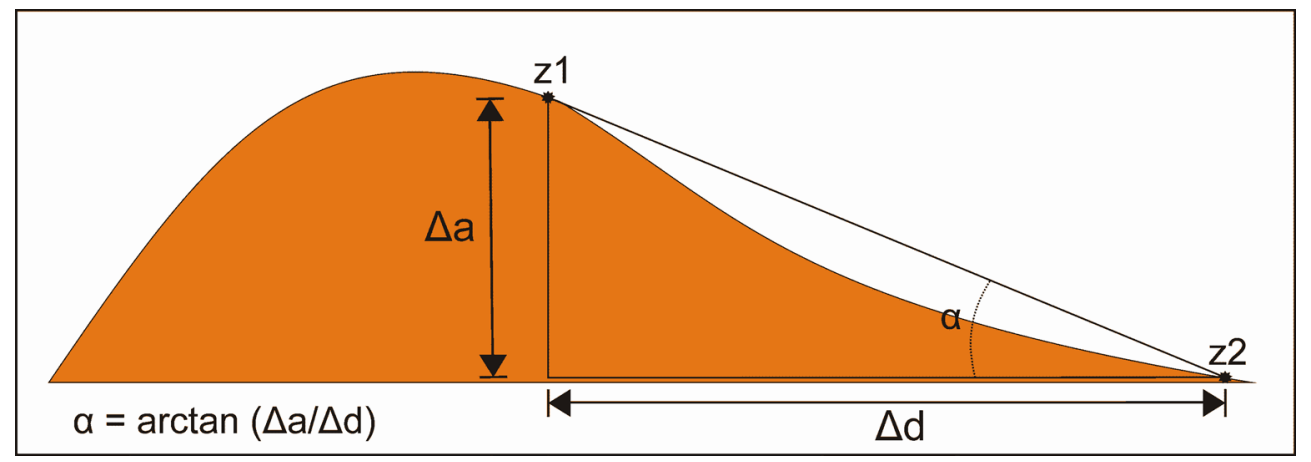

Figura 12. Cálculo de la prominencia de un objeto ubicado en la cota z1 respecto al punto de vista del observador ubicado en la cota z2. La prominencia es el ángulo a.

Para verificar esta hipótesis, he recurrido a un procedimiento sencillo para determinar la posición que ocupa un observador respecto a un elemento que le es visible $^{9}$. Se basa únicamente en determinar el ángulo de elevación existente entre el punto de vista del observador y el objeto observado, en línea con el concepto de "angle of incidence" definido por Higuchi (1983). Conociendo la cota de ambos puntos, y la distancia lineal que los separa, es posible realizar una sencilla operación matemática, como se explica en la Figura 12, que nos devuelve el valor de ese ángulo y, por tanto, esa prominencia. Los valores posibles serán positivos, cuando el punto observado se encuentra por encima del punto desde el que se observa; o negativos, cuando el observador está más alto que el punto observado. El rango de valores teóricamente posibles varía entre $+90^{\circ}$ y $-90^{\circ}$, que se corresponderían respectivamente con un ángulo de visión positivo y negativo totalmente verticales.

Tomando este procedimiento, lo que se ha hecho es calcular ese valor de prominencia desde cada una de las posiciones del entorno desde las que el castro es visible. Lógicamente, fue hecho para cada sector de forma separada, de tal manera que para cada sector se identificaron las posiciones del entorno desde las que es potencialmente visible (información que ya obteníamos en el análisis de visibilización anterior) y se calculó el ángulo de elevación desde cada una de esas posiciones. Para resumir todos esos valores y comparar los sectores entre sí, se determinó el valor de la mediana para cada sector. Para mantener la correspondencia con las fases analíticas anteriores, estos valores se extrajeron tanto para las posiciones visibles en el entorno de 700 metros como para las visibles a larga distancia. El resultado, que se muestra gráficamente en la Figura 13, muestra una fuerte convergencia con los resultados cuantitativos del análisis de visibilización: los sectores del poblado más visibles son también los más prominentes, en la medida en la que son mayoritariamente vistos desde posiciones topográficamente inferiores. Además, esta tendencia se acentúa si nos fijamos únicamente en la visibilización desde la corta distancia $(700 \mathrm{~m})$. 


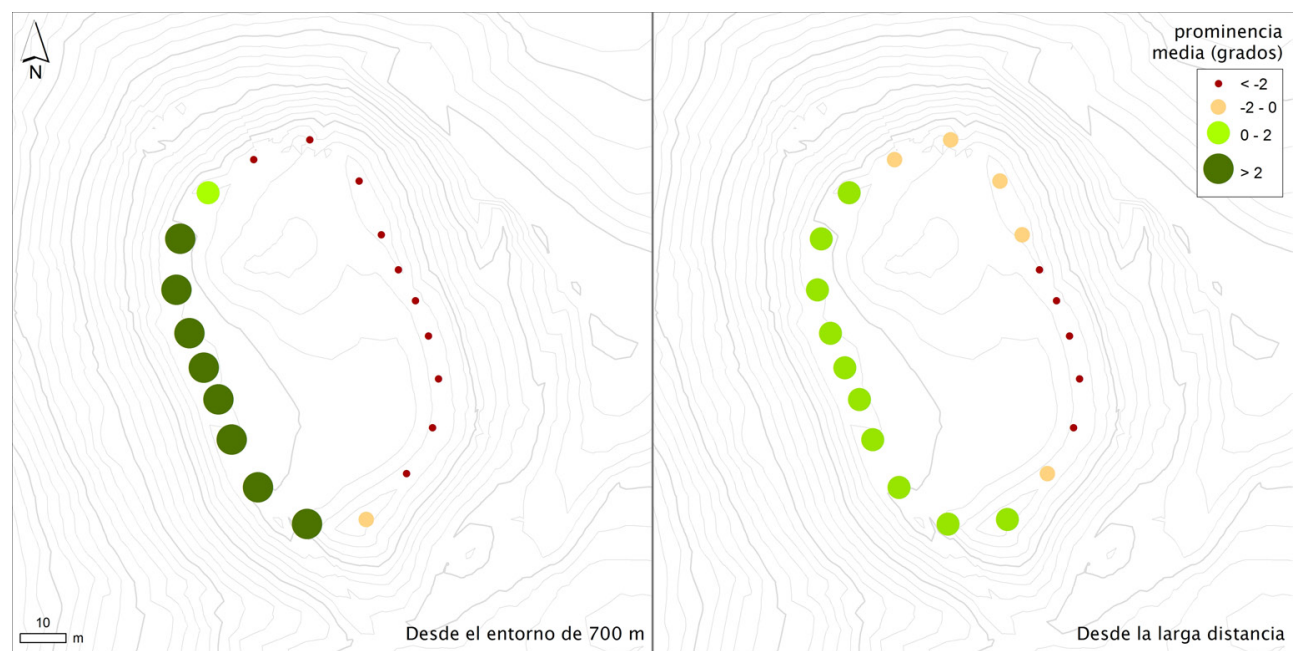

Figura 13. Resultados del análisis de prominencia visual desde el entorno de 700 metros (izquierda) y desde la larga distancia. Los valores muestran el valor de la mediana del conjunto de ángulos con los que cada uno de los sectores es visible desde los lugares en que ese sector se puede ver. El tamaño y tono del punto es proporcional a ese valor: los dos primeros símbolos se corresponden con valores de prominencia negativos, los dos segundos con valores positivos

$\mathrm{Si}$, como hicimos en los pasos anteriores, exploramos la correspondencia entre la variación en estos valores de prominencia y en el volumen de estructuras artificiales, el resultado es el siguiente (para diferentes intervalos de distancia):

Pxy (prominencia en el entorno de $700 \mathrm{~m}, \mathrm{VNMR})=-0,483$

Pxy (prominencia a larga distancia, VNMR) $=-0,516$

Una vez más encontramos una correlación negativa en los términos en los que se ha medido esta relación; es decir, según aumenta la prominencia del perímetro exterior del poblado, disminuye la inversión de esfuerzo en la construcción de estructuras artificiales. En otras palabras, las zonas donde más elementos artificiales se concentran son las menos prominentes topográficamente.

Podría argumentarse que la manera en la que se están manejando los resultados del análisis de prominencia es engañosa. Al considerar como representativo el valor de la mediana de todas las posiciones desde las que es visible cada sector del poblado, podríamos estar introduciendo un sesgo que favoreciese a los sectores que tienen unos valores de prominencia más extremos. Por ejemplo, hacia el Oeste de los sectores 10 a 19 existe una abrupta pendiente inmediata que luego se suaviza pero que hace que haya aquí muchas posiciones deprimidas topográficamente desde las que el castro es visible con un ángulo de incidencia muy acusado.

Para tratar de medir el posible efecto de esto, recurrí a considerar una manera diferente de comprar la variación en la prominencia visual de cada sector. En lugar de comparar las medianas de cada uno, lo que hice fue analizar cada sector de forma independiente (de manera similar a como se hizo anteriormente para la visibilización). Para cada uno de ellos, y conociendo la extensión de las zonas desde las que es visible, se determinó desde qué 


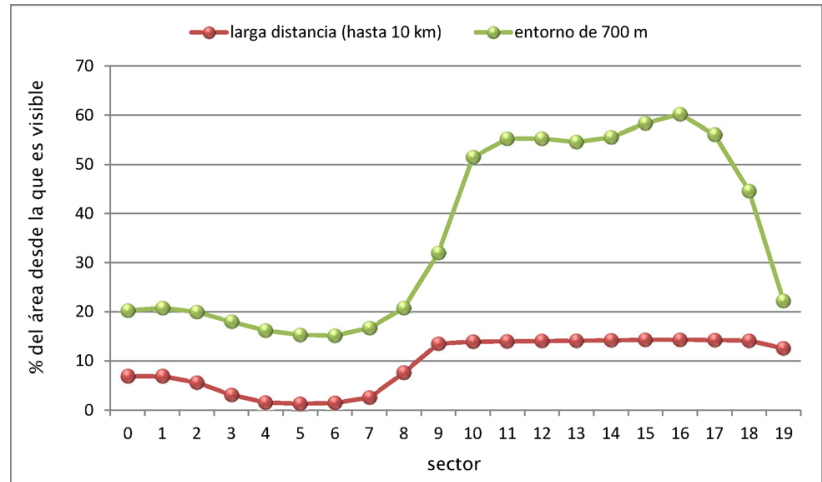

Figura 14. Porcentaje de posiciones desde las que la visibilización hacia cada sector del poblado tiene una prominencia positiva.

porcentaje de esas áreas la prominencia visual era positiva y desde cuál negativa. Tomemos, por ejemplo, el sector 0 . Este sector es potencialmente visible desde un conjunto de posiciones que suman un total de 13,25 km2 en el área de trabajo. Desde 4,37 de esos 13,25 km2, la prominencia visual es positiva; o sea, el 33,01\%. Este porcentaje de posiciones desde las que la prominencia es positiva fue lo que se calculó para cada sector independientemente, y se compara en la Figura 14. En este caso los valores son independientes de la mayor o menor visibilización hacia cada sector, y también de la intensidad de la prominencia, sólo consideran si ésta es positiva o negativa.

Los resultados muestran de nuevo que el conjunto de sectores del 19 al 8 son los que muestran una menor prominencia. Estos sectores, ubicados hacia el Este del poblado, son los mismos que mostraban valores inferiores de prominencia promedio. Esto permite, pues, confirmar la significación de los resultados mostrados anteriormente.

\section{Síntesis}

Los resultados obtenidos permiten considerar de manera diferente las tres hipótesis que se han planteado. Por un lado, los resultados son coherentes con la formulación de la hipótesis 1, ya que efectivamente la inversión de trabajo en la construcción de estructuras artificiales de delimitación del poblado es mayor en las partes topográficamente más accesibles, y desciende en las menos accesibles.

Sin embargo, las otras dos hipótesis, que trataban de explorar la idea de la monumentalización como lógica y criterio locacional y constructivo de los poblados, no son respaldadas por el tipo de indicadores que he analizado. En efecto, las partes del poblado con más volumen de estructuras artificiales no son ni las más visibles (las visibles desde más lugares) ni las "mejor" visibles (las visibles con una mayor prominencia). De hecho, de forma bastante sorprendente, la relación entre ambas cosas (visibilización, ya sea cuantitativa o cualitativa, y construcción de estructuras) es no sólo indiferente sino incluso negativa, ya que precisamente las partes del poblado más artificializadas son las que tienen unas condiciones de visibilización menores y peores. Hay que destacar al respecto que la visibilización se ha medido considerando el volumen actualmente conservado de estructuras artificiales (o sea, he medido cómo de visibles son las murallas y aterrazamientos hoy conservados). Esto 
permite descartar la posibilidad de que las estructuras hayan sido construidas en las zonas menos visibles precisamente para contrarrestar esa carencia, sino que una vez construidas siguen siendo las partes menos y "peor" visibles del poblado.

Hasta aquí lo que la secuencia analítica realizada en Castrolandín permite proponer. A partir de estos resultados sería posible acceder a un nivel de lectura propiamente interpretativo, pero antes de ello es conveniente atender a una nueva incertidumbre: ¿cómo de representativos pueden considerarse los resultados de Castrolandín de algo más que de este caso en particular? Las cuestiones que se plantean en los apartados iniciales de este trabajo son de orden general, pero el análisis se ha centrado en un único caso de estudio. Una manera evidente de explorar la significación de los resultados de este caso es analizando otros.

\section{Resultados obtenidos en otros casos}

La metodología y el procedimiento analítico que he presentado posibilitan el análisis equivalente de cualquier otro caso, únicamente a condición de disponer de unos recursos básicos, tanto en datos como en herramientas. Con la única finalidad ahora de disponer de al menos algún caso de estudio adicional que permitiese contrastar la significación de los resultados de Castrolandín, una versión simplificada de esta misma secuencia de análisis (un único intervalo de distancia) se aplicó a otro castro para el que disponía de información topográfica y estratigráfica adecuada. Se trata de Alto do Castro de Laxos, excavado en el marco de un proyecto de corrección de impacto en los años 90 (ver Cobas y Parcero-Oubiña 2006).

Los resultados de los indicadores principales de correlación en este caso fueron:

Pxy (visibilización, VNMR) $=0,074$

Pxy $($ coste, $\mathrm{VNMR})=-0,798$

Estos valores apuntan en una línea muy similar a Castrolandín: de nuevo hay una correlación negativa elevada entre coste de acceso y volumen de estructuras artificiales, de nuevo las defensas son más y mayores en las partes más accesibles del poblado. En este caso la correlación con la visibilización es mucho más neutra, de hecho es casi nula; esto, en cualquier caso y en relación a las hipótesis planteadas, no cambia el hecho de que la mayor inversión en elementos defensivos siga sin producirse en las partes más visibles del poblado. Todo ello es muy coherente con las semejanzas entre ambos sitios, de hecho son castros muy próximos (distantes apenas $1 \mathrm{~km}$ lineal), similares en dimensiones y, aunque el de Laxos tiene una historia de ocupación más amplia, habrían estado ocupados simultáneamente en gran medida, hacia el final de la Edad del Hierro.

\section{Observaciones finales}

"Evaluating the role of defensiveness and conflict in cultural history is an interpretive step beyond syntactical calculations" (Martindale y Supernant 2011: 202). No hay duda de que una serie de mediciones más o menos prolijas y detalladas no son la respuesta a una cuestión tan compleja y sutil como ésta. De hecho, ni siquiera proporcionan una relación de causalidad indiscutible entre los fenómenos que se han explorado por medio de ellas. En realidad lo único que aporta la secuencia analítica mostrada es una serie de correlaciones, o de ausencia 
de ellas, entre varias variables medidas. Cualquier correlación, por robusta que sea, no puede tomarse como una razón de causalidad sino es a través de un proceso interpretativo posterior. Pero, al tiempo, la ausencia de correlación sí aporta algo más, en la medida en la que hace inviables las hipótesis planteadas, al menos en la forma en la que lo han sido.

El análisis realizado permite proponer con un respaldo empírico que la idea de que la construcción de las estructuras defensivas en estos dos poblados haya seguido una lógica defensiva es plausible. Sin embargo, resulta más difícil mantener la idea de que esas estructuras hayan sido concebidas fundamentalmente como elementos monumentales, al menos en los dos casos analizados. Desde luego esto es una lectura extremadamente simplista, en la que un problema amplio y complejo se ha explorado de manera muy simplificada y esquemática. Pero, al menos, tiene un valor indudable: el de ser "objetiva", en el sentido de soportada por una serie de observaciones empíricas, mensurables, y no puramente una argumentación discursiva (como reclama Ruíz Zapatero 2012: 98).

Sin duda esto no agota, ni mucho menos, las lecturas posibles. De hecho, el propio planteamiento puede resultar engañoso si se entiende que las dos opciones principales que se han explorado, la defensiva y la monumental, se conciben como excluyentes. Como ya se ha señalado varias veces, es razonable pensar que en la mayor parte de los casos sean lecturas complementarias (Armit 2007), con importancia cambiante en el tiempo (Parcero Oubiña 2005). Pero, de nuevo, plantear y explorar estas cuestiones en los términos aquí mostrados al menos proporciona un soporte mensurable y apoyado en evidencia empírica para los niveles más básicos de significado de estos elementos materiales. Tal vez el mayor valor de una aproximación de este estilo esté no en aquello que permite proponer, sino en aquello que permite descartar argumentadamente. Extender este proceso de análisis a un conjunto más amplio de sitios permitiría, además, explorar en qué medida decisiones locacionales de este estilo operan a nivel particular o pueden generalizarse dentro de un ámbito más extenso. Este trabajo se ha centrado en proponer un procedimiento metodológico concreto, accesible, y no en explorar a fondo las posibilidades interpretativas que se puedan derivar de los resultados que ése ofrezca.

En términos más metodológicos, sin duda sería posible mejorar el proceso analítico, de tres formas. La primera sería considerando otros factores locacionales y constructivos de manera similar; un ejemplo posible, entre otros, sería la protección contra agentes climáticos, como el viento. La segunda, diseñando otras formas de considerar los mismos factores aquí manejados; por ejemplo, considerando la visibilización no como un factor isotrópico, igual de relevante en cualquier dirección y desde cualquier punto, sino como algo especialmente pertinente desde algunas posiciones, por ejemplo desde las rutas de acceso al sitio. La tercera sería explorando otros escenarios posibles sobre la evidencia actualmente disponible en este sitio, como reconstrucciones hipotéticas del volumen original de las defensas. Aunque esta opción está limitada por los datos concretos de que se pueda disponer acerca de cada sitio, tiene la notable ventaja de ser muy fácilmente implementable con la metodología propuesta. Sería sencillo diseñar varios escenarios reconstructivos posibles y testar cada una de las hipótesis en esas distintas condiciones, para ver cómo de consistentes son, o como de dependientes de ciertos cambios en las posibles formas originales de la arquitectura de los sitios. 
En cualquier caso, incorporar nuevos factores de este estilo permitiría seguir manteniendo como posibles, o descartar, algunas opciones e hipótesis frente a otras, y sobre todo contribuiría a acotar los límites posibles de la interpretación alrededor de un fenómeno, el de la fortificación, que a menudo es abordado a partir de argumentos ampliamente retóricos, o directamente ad hoc.

Agradecimientos: Patricia Mañana Borrazás hizo el escaneado láser de Castrolandín, y además me ayudó con extrema habilidad y solvencia a manejar los datos derivados de ese trabajo y adaptarlos al uso que le he dado aquí. El procedimiento concreto para el cálculo de prominencia visual es original de Pastor Fábrega Álvarez, quien me ha permitido emplearlo aquí antes de haberlo publicado él mismo.

\section{Notas}

1. Este artículo es una versión extendida de un trabajo que, en diferentes estados de elaboración, fue presentado en 2007 primero como comunicación en un congreso en Durham (UK) y después como póster en un congreso en Mérida (España). Ninguna de esas contribuciones fue publicada.

2 Este trabajo se centra en los argumentos y discusiones desarrolladas en torno al registro del occidente europeo, aunque debates semejantes vienen ocurriendo ciertamente en relación con otros contextos (p.e. Arkush y Stanish 2005, Smith y Cochrane 2011).

3 Siguiendo a Criado Boado (1993), he preferido usar visibilización en lugar de visibilidad para denotar la diferencia entre lo que es visible desde un lugar (visibilidad) y los lugares desde los que algo es visible (visibilización).

4 Ver nota 1.

5 Los parámetros de precisión de este DEM son, para 150 puntos de control: RMS error: 1,334m; Error máximo: 10,455m; Error mínimo: 0,013m. El error máximo es muy elevado pero corresponde a un único punto aislado; la desviación estándar respecto al promedio es de $1,515 \mathrm{~m}$.

6 Para dar una idea del grado de detalle de este TIN, consta de 244.436 triángulos para una superficie total mapeada de 5,27 Has., de lo que resulta un tamaño promedio de los triángulos de apenas $21 \mathrm{~cm}$.

7 Y, también en parte, como fue dicho, de su destrucción una vez abandonado el sitio. Sin embargo, y como se apuntó, no hay evidencias de que esta destrucción haya afectado con diferente intensidad a las distintas partes del sitio. Por ello, las diferencias relativas actualmente visibles en el volumen de las estructuras pueden considerarse representativas de las originales.

8 Este coeficiente es una de las formas más habituales de expresar la correlación entre dos variables, la manera en la que el cambio en ambas es simultáneo o independiente. Se expresa en una magnitud entre 0 (no hay correlación alguna) y 1 (hay una correlación perfecta), y pueden ser positivos (el cambio en ambas variables se da en la misma dirección, ambas aumentan o descienden en paralelo) o negativos (cuando una asciende, la otra desciende). Este índice exige que las variables analizadas sean independientes. Aunque en este caso las observaciones tienen una cierta dependencia, dada su disposición circular (cada punto es probable que se parezca más a los que le rodean que a los otros), las variables analizadas (visibilidad, facilidad de acceso, volumen construido) sí son independientes. Además, esa esperable semejanza por proximidad no se da en todos los casos, pues ocurren cambios abruptos (ver por ejemplo figura 11).

9 Este procedimiento ha sido desarrollado por mi compañero Pastor Fábrega Álvarez en el marco de su trabajo doctoral, y hasta ahora no había sido publicado. 


\section{Bibliografía citada}

Arkush, E. y M.W. Allen (eds.)

2006 The Archaeology of Warfare: Prehistories of Raiding and Conquest. University of Florida Press, Gainesville.

Arkush, E., y C. Stanish

2005 Interpreting Conflict in the Ancient Andes. Current Anthropology 46(1): 3-28.

Armit, I.

2007 Hillforts at War: From Maiden Castle to Taniwaha Pā. Proceedings of the Prehistoric Society 73: 25-38.

Ayán Vila, X. (ed.)

2002 Pasado e futuro de Castrolandín (Cuntis): Unha proposta de recuperación e revaloración. TAPA

29. Instituto de Estudos Galegos Padre Sarmiento, Santiago de Compostela.

Ayán Vila, X., C. Otero Vilariño y A. González Ruibal

2007 Sondaxes arqueolóxicas no castro de Castrolandín (Cuntis, Pontevedra): campaña 2004.

El Museo de Pontevedra 61: 11-62.

Bevan, A.

2011 Computational models for understanding movement and territory. Tecnologías de Información Geográfica y Análisis Arqueológico del Territorio (ed. por V. Mayoral Herrera y S. Celestino Pérez), pp. 383-394. Anejos de Archivo Español de Arqueología, Mérida.

Connelly, J. y M. Lake

2009 Sistemas de Información Geográfica aplicados a la arqueología. Bellaterra, Barcelona.

Criado Boado, F.

1993 Visibilidad e interpretación del registro arqueológico. Trabajos de Prehistoria 50: 39-65.

Criado Boado, F. y X. Ayán Vila

2006 Sondaxes arqueolóxicas no castro de Castrolandín (Cuntis, Pontevedra): informe valorativo. El Museo de Pontevedra 60: 11-82.

Fairén, S.

2004 ¿Se hace camino al andar? Influencia de las variables medioambientales y culturales en el cálculo de caminos óptimos mediante SIG. Trabajos de Prehistoria 61(2): 25-40.

González García, F.J.

2006 El Noroeste de la Península Ibérica en la Edad del Hierro: ¿una sociedad pacífica? Cuadernos de Estudios Gallegos LIII(119): 131-155.

González García, F.J. (ed.)

2007 Los pueblos de la Galicia céltica. Akal, Madrid.

González Ruibal, A.

2008 Los pueblos del Noroeste. De Iberia a Hispania (ed. por F. Gracia Alonso), pp. 899-930. Ariel, Barcelona. 
Guilaine, J. y J. Zammit

2002 El Camino de la Guerra: La Violencia en la Prehistoria. Ariel, Barcelona.

Higuchi, T.

1983 Visual and Spatial Structure of Landscapes. MIT, Massachusetts.

Howey, M.C.L.

2007 Using multi-criteria cost surface analysis to explore past regional landscapes: a case study of ritual activity and social interaction in Michigan, AD 1200-1600. Journal of Archaeological Science 34(11): 1830-1846.

Keeley, L.H., M. Fontana y R. Quick

2007 Baffles and Bastions: The Universal Features of Fortifications. Journal of Archaeological Research 15: 55-95.

Llobera, $\mathrm{M}$.

2001 Building Past Landscape Perception With GIS: Understanding Topographic Prominence. Journal of Archaeological Science 28(9): 1005-1014.

2007 Reconstructing visual landscapes. World Archaeology 39(1): 51-69.

Llobera, M., P. Fábrega Álvarez y C. Parcero Oubiña

2011 Order in movement: a GIS approach to accessibility. Journal of Archaeological Science 38(4): 843-851.

Mañana Borrazás, P., R. Blanco Rotea y A. Rodríguez Paz

2009 La documentación geométrica de elementos patrimoniales con láser escáner terrestre. La experiencia del LaPa en Galicia. Cuadernos de Estudios Gallegos 122: 33-65.

Martindale, A. y K. Supernant

2009 Quantifying the defensiveness of defended sites on the Northwest Coast of North America. Journal of Anthropological Archaeology 28(2): 191-204.

Murrieta Flores, P.

2012 Understanding human movement through spatial technologies. The role of natural areas of transit in the Late Prehistory of South-western Iberia. Trabajos de Prehistoria 69(1): 103-122.

Otero, I., E. Varela, S. Mancebo y A. Ezquerra

2009 El análisis de visibilidad en la evaluación de impacto ambiental de nuevas construcciones. Informes de la Construcción 61(515): 67-75.

Otero Vilariño, C. y Y. Porto Tenreiro

2007 Castrolandín: el proceso de empoderamiento en un proyecto de gestión cultural. Actas IV Congreso Internacional sobre Musealización de Xacementos Arqueolóxicos: conservación e presentación de xacementos arqueolóxicos no medio rural, pp. 377-383. Xunta de Galicia, Santiago de Compostela.

Parcero Oubiña, C.

2000 Tres para dos. Las formas del poblamiento en la Edad del Hierro del Noroeste Ibérico. Trabajos de Prehistoria 57(1): 75 - 95. 
2005 Variaciones en la función y sentido de la fortificación durante la Edad del Hierro del Noroeste. Bronce Final y Edad del Hierro en la Península Ibérica. Encuentro de Jóvenes Investigadores (ed. por A. Blanco, C. Cancelo y Á. Esparza), pp. 11-33. Universidad de Salamanca, Salamanca.

Parcero Oubiña, C. y P. Fábrega Álvarez

2006 Diseño metodológico para el análisis locacional de asentamientos a través de un SIG de base raster. Territorios Antiguos y Nuevas Tecnologías. La aplicación de los SIG en la Arqueología del Paisaje (ed. por I. Grau Mira), pp. 69-91. Universidad de Alicante, Alicante.

Rahn, B.

2005 Cost Surface-Derived Least-Cost Paths: A Case Study from Iron Age Orkney. Internet Archaeology 19.

Ruíz Zapatero, G.

2012 Settlement and Landscape in Iron Age Europe: Archaeological Mainstreams and Minorities. Atlantic Europe in the First Millennium BC: Crossing the Divide (ed. por T. Moore y X.L. Armada Pita), pp. 81-108. Oxford University Press, Oxford.

Sevenant, M. y M. Antrop

2007 Settlement models, land use and visibility in rural landscapes: Two case studies in Greece. Landscape and Urban Planning 80(4): 362-374.

Smith, C. y E.E. Cochrane

2011 How is visibility important for defence? A GIS analysis of sites in the western Fijian Islands. Archaeology in Oceania 46: 76-84.

Tobler, W.

1993 Three presentations on geographical analysis and modelling. National Center for Geographic Information and Analysis, Santa Barbara.

Verhagen, P. y T. Whitley

2012 Integrating Archaeological Theory and Predictive Modeling: a Live Report from the Scene. Journal of Archaeological Method and Theory 19: 49-100.

Wheatley, D. y M. Gillings

2000 Vision, Perception, and GIS: developing enriched approaches to the study of archaeological visibility. Beyond the Map: Archaeology and spatial technologies (ed. por G. Lock), pp. 1-27. IOS Press, Amsterdam. 